\title{
How close are we to preventing prostate cancer?
}

\author{
Peter T Scardino
}

Some years ago the legendary Paul Peters, then Secretary of the American Urological Association (AUA), liked to taunt his audience, warning that urologists sit on a three-legged stool-prostate cancer, BPH and bladder cancer-only three drugs away from oblivion as a surgical specialty. While there are no such drugs for bladder cancer, drug therapy for BPH has had a profound impact on urological practice. Alpha-blockers and 5- $\alpha$ reductase inhibitors (5aRls) allow most patients to delay or completely avoid invasive therapy. Transurethral resection of the prostate, once the most common operation performed by urologists in the US, fell from 258,000 procedures per year in 1987 to 87,000 in 2000.

There are no comparable oral medications to treat early prostate cancer. Although complete androgen ablation or antiandrogens delay progression, they do not eliminate cancer and have no survival benefit. We may, however, be closer than we realize to preventing prostate cancer.

In the landmark Prostate Cancer Prevention Trial (PCPT), finasteride reduced the risk of developing prostate cancer by $25 \%$ compared with placebo. Enthusiasm was dampened, however, by the $1.3 \%$ absolute increase in the risk of high-grade cancer in the finasteride arm of the trial. As a result, few have embraced the benefits of finasteride, and some men taking $5 \alpha \mathrm{Rls}$ for either baldness or BPH worry that they should stop the medication.

Data presented at recent national scientific meetings support the concept that $5 \mathrm{aRls}$ may be safe and effective in preventing prostate cancer. In an elegant study presented at the Canadian Urological Association meeting, prostate size was shown to be a significant factor in detecting high-grade cancers on needle biopsy. High-grade cancer was significantly more likely in needle biopsies from smaller (compared with larger) prostates. At the AUA meeting, a similar result was reported after the PCPT data were reanalyzed. Finasteride reduced the size of the prostate by $20-30 \%$, yet the same number
Chemo-

prevention

of prostate

cancer is at

hand. The

implications

for the

management

of this

disease are

as profound

as those

of medical

therapy for

$\mathrm{BPH}$,

PT Scardino is the

Editor-in-Chief

of Nature Clinical

Practice Urology,

Chairman of the

Department of

Urology at Sidney

Kimmel Center for

Prostate and Urologic

Cancers, and Alfred

$P$ Sloan Chair at

Memorial Sloan-

Kettering Cancer

Center.

\section{Competing interests}

The author declared competing interests; go to

the article online for details.

www.nature.com/clinicalpractice doi:10.1038/ncpuro0256 of biopsy cores per prostate were taken from both finasteride and placebo groups. If $5 \mathrm{aRls}$ have no effect on high-grade tumors but prevent or inhibit low-grade lesions, we would expect an overall reduction in cancer risk with a slight increase in high-grade lesions, precisely what the PCPT originally reported. When the results were adjusted for prostate volume, the increased risk of high-grade cancer disappeared and the reduction in overall cancers was even greater.

In the past year, several studies have reported that cholesterol-lowering statins also reduce the risk of developing or of dying from prostate cancer. These drugs must be tested prospectively, in well-controlled trials, before we recommend their use for prostate cancer prevention, but the mechanism makes sense. Dietary fat-and, to a lesser degree, total caloric intake and BMIhave long been implicated as risk factors for dying from prostate cancer.

Other hormonal agents show promise in the prevention of prostate cancer. At the AUA and American Association for Cancer Research meetings, toremifine, a selective estrogen-receptor modulator, was reported to reduce the risk of progression from prostatic intraepithelial neoplasia to prostate cancer. A large trial of dutasteride is underway, supported by GlaxoSmithKline. Micronutrients-vitamin $E$ and selenium-are being tested in the large, randomized National Cancer Institutesponsored SELECT trial, which has completed accrual of over 32,000 men and is scheduled to report the first interim analysis in 2006.

Chemoprevention of prostate cancer is at hand. The implications for the management of this disease are as profound as those of medical therapy for BPH. We seem poised to saw off the second leg of Paul Peters' three-legged urological stool. Surely, lifesaving radical surgery and radiation therapy for prostate cancer will persist, but the need is likely to fall substantially as chemoprevention is established and embraced. Paul Peters, how right you were! 